\title{
Gut resistome development in healthy twin pairs in the first year of life
}

\author{
Aimee M. Moore ${ }^{1,3}$, Sara Ahmadi ${ }^{2,3}$, Sanket Patel ${ }^{2,3}$, Molly K. Gibson ${ }^{3}$, Bin Wang 2,3, I. Malick Ndao ${ }^{1}$, Elena Deych
} William Shannon ${ }^{4}$, Phillip I. Tarr ${ }^{1,5}$, Barbara B. Warner ${ }^{1}$ and Gautam Dantas ${ }^{2,3,6^{*}}$

\begin{abstract}
Background: The early life of the human host marks a critically important time for establishment of the gut microbial community, yet the developmental trajectory of gut community-encoded resistance genes (resistome) is unknown. We present a longitudinal study of the fecal antibiotic resistome of healthy amoxicillin-exposed and antibiotic-naive twins and their mothers during the first year of life.

Results: We extracted metagenomic DNA (mgDNA) from fecal samples collected from three healthy twin pairs at three timepoints (1 or 2 months, 6 or 7 months, and 11 months) and from their mothers (collected at delivery). The mgDNA was used to construct metagenomic expression libraries in an Escherichia coli host. These libraries were screened for antibiotic resistance, and functionally selected resistance genes were sequenced and annotated. A diverse fecal resistome distinct from the maternal resistome was apparent by 2 months of age, and infants' fecal resistomes included resistance to clinically important broad-spectrum beta-lactam antibiotics (e.g., piperacillin-tazobactam, aztreonam, cefepime) not found in their mothers. Dissemination of resistance genes among members of a given family was positively correlated with sharing of those same resistance genes between unrelated families, potentially identifying within-family sharing as a marker of resistance genes emerging in the human community at large. Finally, we found a distinct developmental trajectory for a community-encoded function: chloramphenicol resistance. All study subjects at all timepoints harbored chloramphenicol resistance determinants, but multidrug efflux pumps (rarely found in mothers) were the primary effectors of chloramphenicol resistance in young infants. Chloramphenicol acetyltransferases were more common in mothers than in infants and were found in nearly all the infants at later timepoints.
\end{abstract}

Conclusions: Our results suggest that healthy 1-2-month-old infants' gut microbes harbor clinically relevant resistance genes distinct from those of their mothers, and that family-specific shared environmental factors early in life shape resistome development.

Keywords: Antibiotic resistance, Gut microbiome, Fecal microbiome, Pediatrics, Beta lactamase, Chloramphenicol resistance

\section{Background}

The human gut microbiota is an important reservoir of antibiotic resistance genes (resistome), which can be exchanged with pathogens [1-4]. Diverse antibiotic resistance genes are present in the gut microbiota of young infants soon after birth, even without antibiotic therapy

\footnotetext{
* Correspondence: dantas@wustl.edu

${ }^{2}$ Department of Pathology and Immunology, Washington University in St. Louis School of Medicine, 660 S. Euclid Avenue, St. Louis, MO 63110, USA

${ }^{3}$ Center for Genome Sciences and Systems Biology, Washington University in St. Louis School of Medicine, 4444 Forest Park Boulevard, St. Louis, MO 63108, USA

Full list of author information is available at the end of the article
}

[5-12]. While the overall architecture of the gut microbial community undergoes chaotic shifts in the first years of life before stabilizing towards an adult-like state [13-16], the trajectory and dynamics of resistome development in healthy young infants are poorly understood. Children in the United States are heavily antibioticexposed [11, 12], with unclear effects on resistome development. In this study, we present a longitudinal functional metagenomic interrogation of gut resistome development during the first year of life in three pairs of healthy, vaginally delivered, formula-fed twins enrolled in the St. Louis Neonatal Microbiome Initiative. One pair was antibioticnaïve for the entire sampling interval, one pair was

\section{Biomed Central}

(c) 2015 Moore et al. This is an Open Access article distributed under the terms of the Creative Commons Attribution License (http://creativecommons.org/licenses/by/4.0), which permits unrestricted use, distribution, and reproduction in any medium, provided the original work is properly credited. The Creative Commons Public Domain Dedication waiver (http:// creativecommons.org/publicdomain/zero/1.0/) applies to the data made available in this article, unless otherwise stated. 
concordant (receiving 10 days of amoxicillin simultaneously at 8 months of age) and one was discordant (one sibling received 10 days of amoxicillin at 8 months and the other did not).

\section{Results}

Demographic information regarding the three families included in this study is listed in Additional file 1: Table $\mathrm{S} 1$. We constructed 21 fecal metagenomic libraries averaging $9.3 \pm 7.9$ (mean \pm s.d.) gigabases $(\mathrm{Gb})$ in size, from 18 infant samples and 3 maternal samples, and used functional metagenomic selections paired with nextgeneration sequencing to identify a total of 905 unique predicted antibiotic resistance protein clusters (collapsed at $97 \%$ amino acid identity using cd-hit $[17,18])$. In all individuals, at all timepoints, we identified protein clusters conferring resistance to five different antibiotic classes $(\beta-$ lactams, tetracycline, chloramphenicol, trimethoprim, and cycloserine). Proteins conferring resistance to aminoglycosides, tigecycline, and colistin were also found, but less frequently. Of the 905 resistance protein clusters identified, 296 (32 \%) were unique to mothers, 531 (59 \%) were unique to infants, $142(15.6 \%)$ were shared between twin siblings, 159 (17.5\%) persisted within the same individual at multiple timepoints, and $106(11.7 \%)$ were shared between unrelated infants. Only 51 (5.6\%) were shared between infants and their mothers. Twin siblings' resistomes were significantly more similar to each other than to those of their mothers or unrelated infants $(p<0.01$, Student's $t$-test with 1000 Monte Carlo simulations, Fig. 1). Conversely, they were as similar to their siblings' resistomes as they were to their own at other points in time and no more similar to their mothers' resistomes than to unrelated infants' resistomes (Fig. 1). There was no difference in sharing between infants and their own mothers and infants and unrelated mothers. When sharing of resistance proteins between siblings was separated by individual timepoint, infant resistomes were significantly more similar to their twin sibling's resistome than to the resistome of an unrelated infant $(p=<0.005$, all timepoints, Additional file 1: Figure S3). The composition of infant resistomes appears generally robust in the face of amoxicillin perturbation: neither the overall population of resistance protein nor the specific sub-population of $\beta$-lactamases was affected by amoxicillin exposure when measured approximately 3 months after antibiotic treatment. There was, however, a statistically significant increase in the number of multidrug efflux pumps

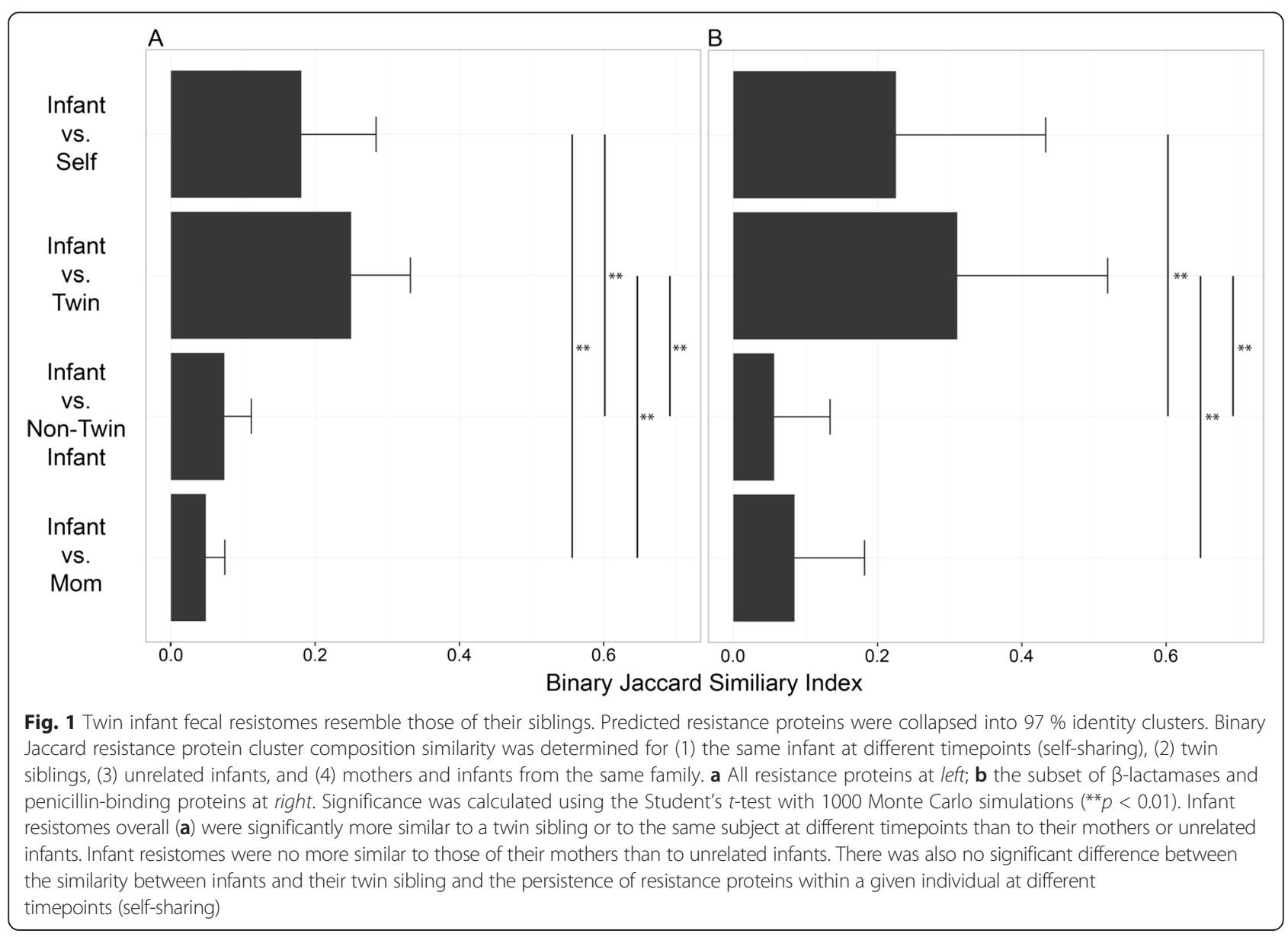


conferring resistance to $\beta$-lactam antibiotics at 3 months after amoxicillin exposure in these infants $(p<0.0001$, loglinear model, Additional file 1: Table S3).

We used a log-linear model to test the likelihood that resistance-associated protein clusters would be shared within multiple members of a given family, or between unrelated families (Fig. 2). Although resistance-associated proteins were less likely to be found in more than one family member than in just one individual $(p<0.0001$, Additional file 1: Table S4) and less likely to be found in multiple families than in a single family $(p<0.0001$, Additional file 1: Table S4), resistance proteins that were found in more than one individual within a family were also significantly more likely to be found in more than one family $(p<0.0001$, Additional file 1 : Table S4). We also used a log-linear model to test the likelihood that resistance-associated protein clusters that persisted within a given individual at different timepoints were more likely to be shared within and between families. Although resistance proteins were significantly less likely to be persistent at multiple timepoints than found at a single timepoint $(p<0.0001$, Additional file 1: Table S10), and also less likely to be found in multiple individuals than in a single individual $(p<0.0001$, Additional file 1: Table S10), resistance proteins that persisted within an individual over time were also significantly more likely to be
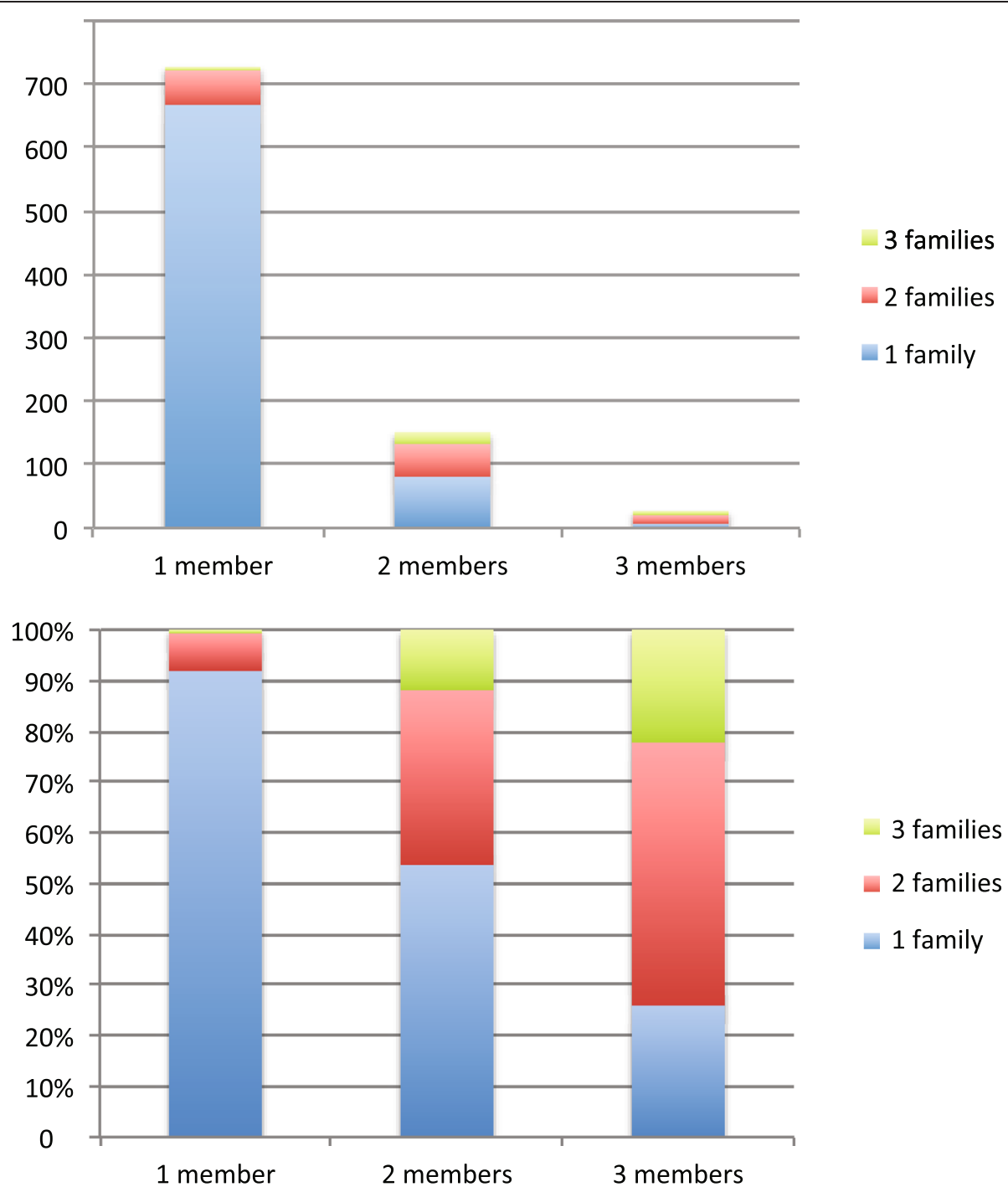

Fig. 2 Sharing of resistance-associated proteins within and between families. The top graph shows absolute counts of resistance-associated protein clusters, grouped by the number of members within a given family they were identified in. Most protein clusters were only identified in one member of one family; much smaller numbers were identified in multiple family members or multiple families. The lower graph shows the proportions of resistance-associated protein clusters identified in multiple families, grouped by the number of members of a single family they were identified in. Larger proportions of resistance-associated proteins that are shared are multiple members of a single family are also identified in multiple unrelated families 
found within multiple members of the same family ( $p=$ $0.0449)$. There was no significant interaction between persistence of resistance proteins within an individual over time and sharing between unrelated families.

We used a log-linear model to test the contribution of co-localization with a mobile genetic element to sharing of resistance protein clusters within members of a single family and between unrelated families. One hundred thirty-six of 905 (15.1 \%) resistance-associated protein clusters were encoded by genes co-localized with a mobile genetic element. However, this co-localization was not significantly associated with sharing of a protein cluster within members of a given family $(p=0.2212$, Additional file 1: Table S5) or between members of unrelated families ( $p=0.2655$, Additional file 1: Table S5).

$\beta$-lactam resistance was universal across the study cohort, yet there was substantial inter-subject variation in the pattern of specific $\beta$-lactam resistance phenotypes (Figs. 3 and 4 and Additional file 1: Figures S1 and S2).
Only 5 of $47(10.7 \%) \beta$-lactamase family proteins were shared between infants and mothers, similar to the proportion shared with unrelated infants (4 of 47 , or $8.5 \%$ ). Consistent with trends observed in the general resistome, $\beta$-lactam resistance patterns were similar between siblings and stable over time, where 11 of 47 (23.4\%) $\beta$ lactamases and penicillin-binding proteins were shared with the infants' twin sibling and 13 of 47 (27.7\%) persisted within the same individual at multiple samplings. Infant $\beta$-lactam resistomes were significantly more like their own resistome at another timepoint or a twin sibling's resistome than the resistome of an unrelated infant, and significantly more similar to their twin siblings' than to their mothers' resistomes $(p<0.01$, Student's $t$-test with 1000 Monte Carlo simulations, Fig. 1b). When each timepoint was analyzed separately, twin siblings' resistomes were only significantly more similar to each other than to those of an unrelated infant of the same age at timepoint 2 (Additional file 1: Figure S3).

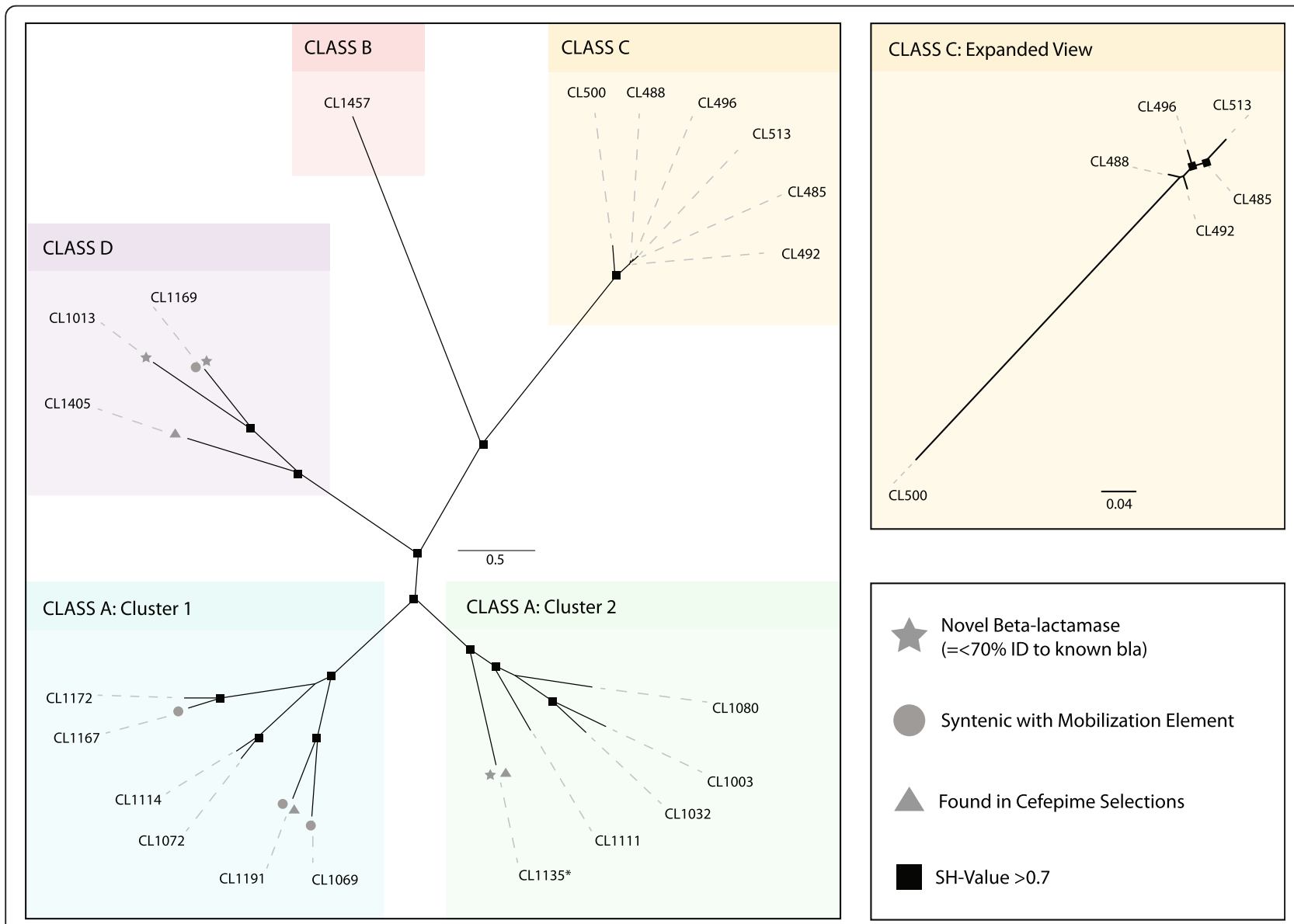

Fig. $3 \beta$-lactamase phylogenetic tree. Predicted $\beta$-lactamase protein sequences were collapsed into $97 \%$ ID clusters. All $\beta$-lactamase protein sequences with at least $90 \%$ coverage of the nearest hit in the NCBI nr database were included in the tree. Multiple alignment was done with Muscle and the tree was made using FastTree. Nodes with an S-H value $>0.7$ are marked with a square. All classes of $\beta$-lactamases are present. Class A $\beta$-lactamases separated into two groups: one with high identity to TEM extended-spectrum $\beta$-lactamases and one without. $\beta$-lactamases co-localized with mobile genetic elements are marked with a gray dot. Novel $\beta$-lactamases with less than $70 \%$ identity to any known $\beta$-lactamase are marked with a star. $\beta$-lactamases found in cefepime selections are marked with a triangle 


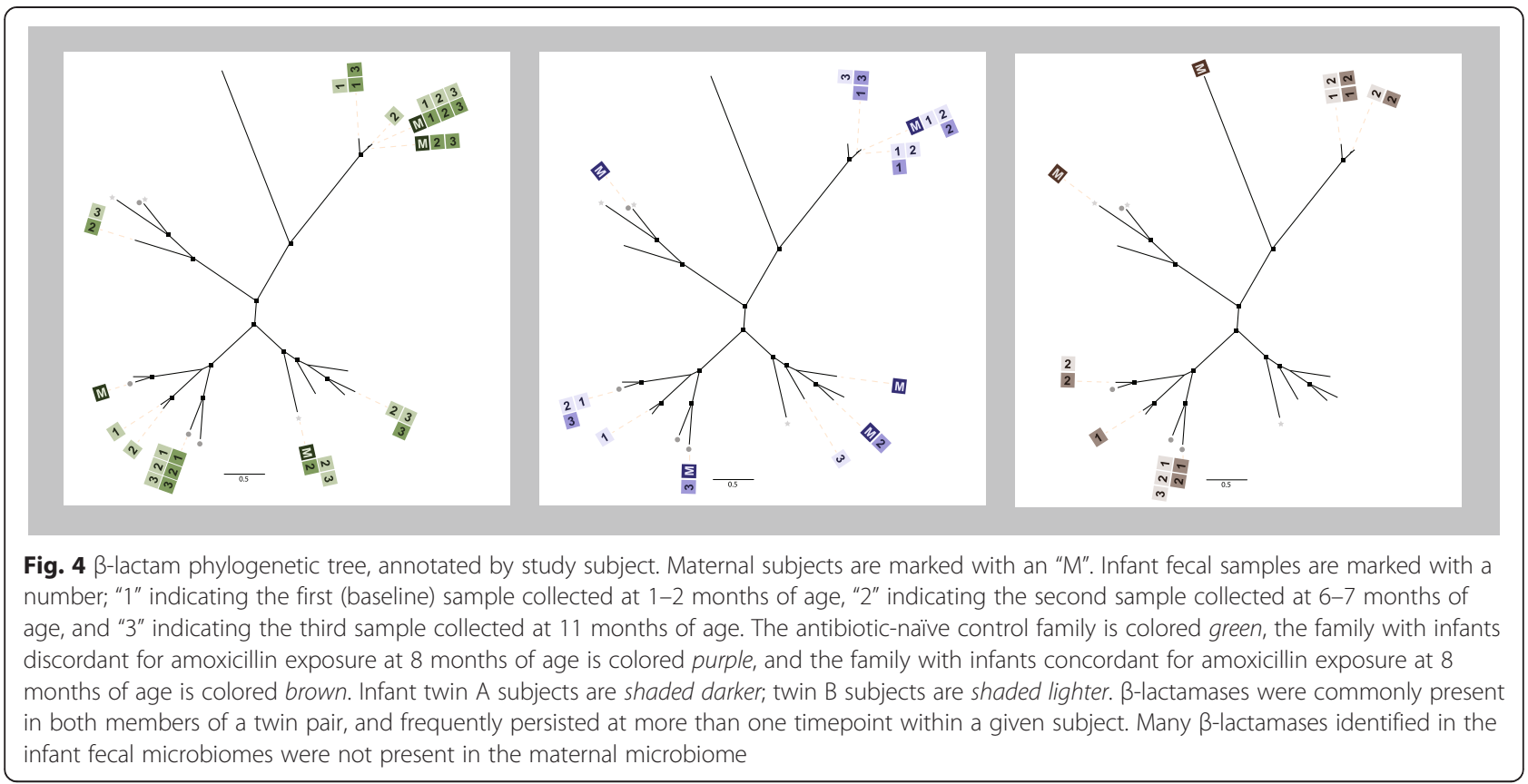

Chloramphenicol resistance was present in all subjects at all timepoints. We observed distinct infantile and adult-like populations of chloramphenicol resistance genes. In the fecal samples collected in early infancy ( 1 or 2 months, and 6 or 7 months), chloramphenicol resistance genes primarily encoded multidrug efflux pumps (Fig. 2). We used a log-linear model to test the likelihood that multidrug efflux pumps were found in mothers versus infants and that these pumps became less common over time. Multidrug efflux pumps were significantly more common in infants than in mothers ( $p=0.0009$, Additional file 1: Table S6) and were significantly less likely to be found as infant age increased during the first year of life $(p<0.0001$, Additional file 1 : Table S7). A majority ( $85 \%$ ) of these efflux pumps had $>90 \%$ identity to efflux pumps of Enterobacteriaceae origin; only one (CL2137) was identical to a bifidobacterial major facilitator transporter (WP_003817038). Some multidrug efflux pumps originated from source contigs with sequences identical to those of bona fide pathogens: a $3737 \mathrm{bp}$ contig from a 1-month-old infant (and a 1460 bp contig from the twin sibling) had $100 \%$ sequence identity to the recently described pan-resistant Enterobacter aerogenes strain EA1509E [19].

While multidrug efflux pumps were the most common effectors of chloramphenicol resistance in stools of young infants, chloramphenicol acetyltransferases were more frequently associated with maternal resistomes. We used a log-linear model to test the likelihood that chloramphenicol acetyltransferases were found in mothers versus infants and that these acetyltransferases became more common over time. Chloramphenicol acetyltransferases were significantly more common in mothers than infants $(p<0.0001$, Additional file 1: Table S8), were rarely found in the youngest infants, but were found in five of the six infants at the final (11-month) timepoint (Fig. 4). There was, however, no significant increase over time in the likelihood of finding chloramphenicol acetyltransferases in infant gut microbiomes $(p=0.2930$, Additional file 1 : Table S9). Twenty-five percent of the chloramphenicol acetyltransferases identified in this study population were co-localized with mobile genetic elements. Novel proteins made up more than half of the chloramphenicol acetyltransferases identified in this study: seven of the 12 chloramphenicol acetyltransferase clusters identified had $<75 \%$ protein identity to any known acetyltransferase. The source contigs for these novel resistance proteins also had low identity to any known organism.

\section{Discussion}

In this study population, we found a diverse fecal resistome established within 1-2 months of birth. Prior microbiome studies of twin infants have shown that siblings' microbiomes undergo similar changes over time [13] and are similar to each other irrespective of zygosity $[15,20,21]$; the patterns of sharing of resistance-associated proteins between twin siblings we observed are consistent with this prior work on the overall microbial community, and likely reflect similarities between siblings in microbial community composition. We also found that infants' gut resistomes differed from those of their mothers in early life, which is also consistent with studies showing that the gut microbiota of twin infants are more similar to each other than to their mothers' microbiota [20] and that infant gut- 
associated tetracycline resistance genes $[6,7]$ are distinct from those in the maternal fecal microbiota [6,7]. The assembly of a diverse gut resistome within the first 2 months of life, and the greater resemblance of infant gut resistomes to their own and their siblings' resistomes at other timepoints than to the resistomes of unrelated infants, suggest that family-specific shared environmental factors early in life shape resistome development. The dissimilarity of infant resistomes to those of their mothers suggests that, although gut microbes may be shared between the mothers and vaginally delivered infants [22], the maternal gut resistome is not the primary driver of gut resistome establishment in infants. The previously observed pattern in which twin siblings' microbiomes are similar to each other and dissimilar to their mothers' microbiome also applies to the gut resistome, suggesting that the infants' gut resistome is likely a reflection of community composition.

In every family, phenotypic resistance to at least one broad-spectrum $\beta$-lactam encoded by an infant gut resistome was not observed in the mothers' resistome, although the specific antibiotics involved (piperacillin-tazobactam, cefotaxime, aztreonam, and/or cefepime) varied (Figs. 3 and 5 and Additional file 1: Figure S1). The genetic dissimilarity between infants' beta-lactam resistomes and those of their mothers likely explains this phenotypic difference, as phylogenetic groups of resistance genes were often associated with specific $\beta$-lactam antibiotics. For example, one group of class A $\beta$-lactamases with high identity to TEM $\beta$ lactamases was associated with extended-spectrum penicillin and $\beta$-lactamase inhibitor resistance (Fig. 3 and Additional file 1: Figure S2), while another class A group was more closely associated with resistance to later-generation cephalosporins. The origin of the broader-spectrum beta-lactam resistance in infant gut resistomes is unclear, although colonization with antibiotic-resistant hospital bacterial strains is a possibility that warrants further study (all newborn subjects were healthy hospital-born infants). However, we wish to note that the multidrug-resistant pathogen Escherichia coli ST131 does not colonize the infants in this cohort until well after discharge from the hospital [23]. The lack of time-dependent effects on $\beta$-lactam resistance genes

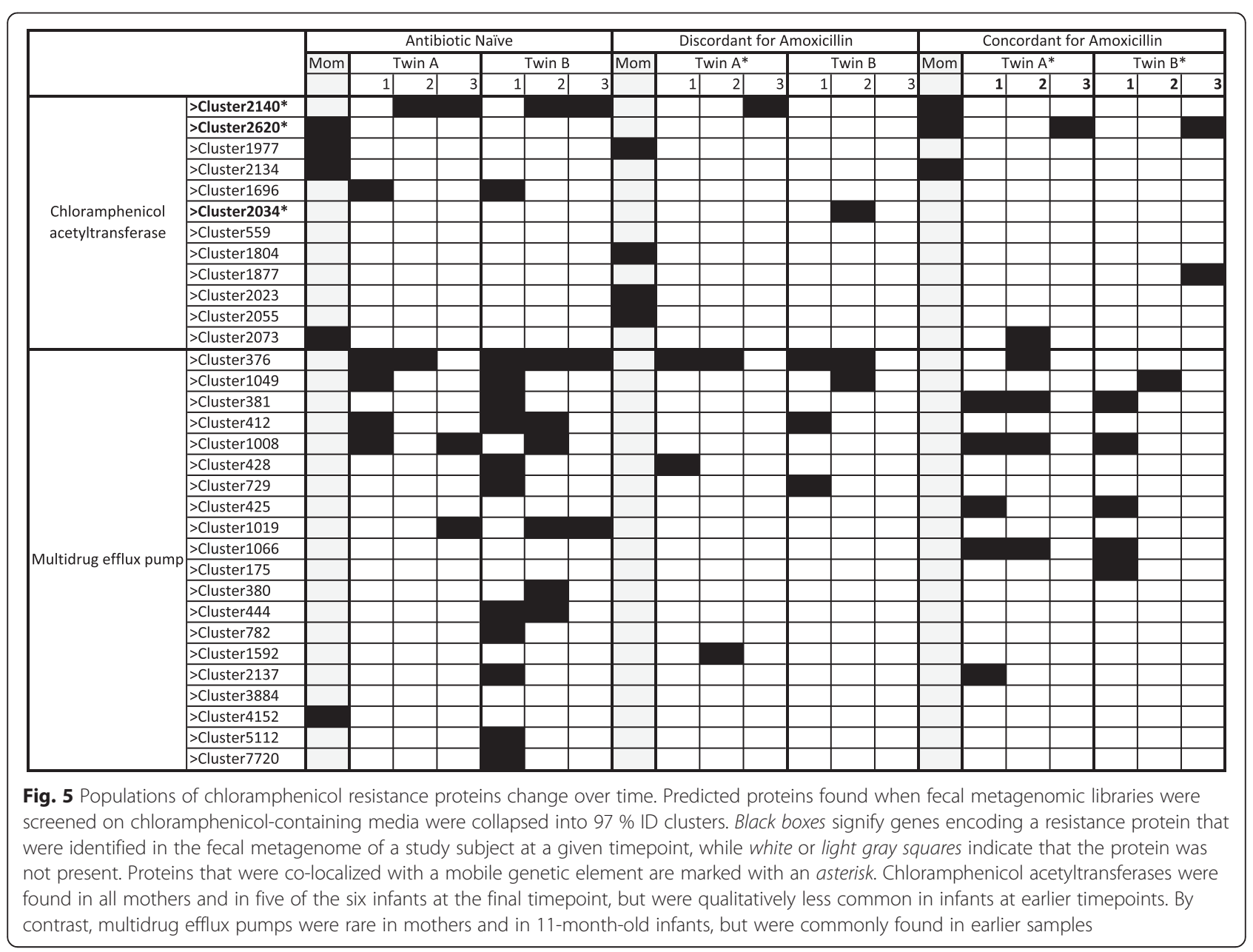


despite inter-subject variation in exposure to amoxicillin may reflect the sampling resolution in this small study, in which the resistome is sampled 1 or 2 months prior to antibiotic therapy and 3 months after antibiotic exposure. Prior studies have shown acute antibiotic-induced perturbations in adult gut microbial community concomitant with host antibiotic exposure, followed by partial recovery $[24,25]$. The binary data regarding presence or absence of resistance genes generated by this study also precludes detection of any changes in the relative abundance of certain taxa following antibiotic exposure. Future studies including more frequent fecal sampling surrounding the time of host antibiotic exposure and additional data regarding the abundance of selected resistance genes may illuminate more acute and transient antibiotic effects that our study was not designed or powered to detect.

Understanding patterns of gut-associated resistance gene dissemination within human communities has epidemiologic implications. We found that sharing of resistance genes within families was positively associated with sharing between families, and that persistence of resistance genes within an individual over time was positively associated with sharing with within-family sharing, but not with between-family sharing. Co-localization with a mobile genetic element was not significantly correlated with identification of resistance proteins in multiple individuals. This finding may be explained by undercounting mobilizable elements in this functional metagenomic study, where nearly all contigs identified were $<5$ kilobases $(\mathrm{kb})$. This concern is partially mitigated by the demonstration that trends in resistance gene and mobilization element co-localization predicted by smallinsert functional metagenomics are recapitulated by matched calculations from bacterial whole genome sequences [26]. Alternatively, our data may suggest that the distribution of gut-associated resistance genes primarily reflects the prevalence of specific host bacteria within the human community, rather than any association with mobile genetic elements, as has been demonstrated in soil microbiomes and resistomes [26]. The positive association between persistence within individuals over time and within-family sharing but not between-family sharing may be a reflection of host genetics or shared environment shaping microbial communities; microbes that are more likely to persist within an individual may also be more likely to colonize close relatives. The potential role for examining genes shared within families to detect clinically important resistance genes emerging human communities warrants further investigation.

Chloramphenicol resistance was universal in all subjects at all timepoints, consistent with our previous resistome study of single timepoint samples of unrelated infants and children [5] as well as with prior studies showing highly prevalent chloramphenicol resistance in adult populations [27]. The reason for universal chloramphenicol resistance is unclear. Agricultural use of chloramphenicol providing selection pressure (the "farm to fork" model [27]) is a potential factor; alternatively, chloramphenicol resistance genes may play a yet-to-be determined role as a core functional component of the gut microbiome [20]. Chloramphenicol resistance genes demonstrated a developmental progression from a neonatal to an adult-like state. The infant chloramphenicol resistome was initially dominated by multidrug efflux pumps, which were uncommonly found in maternal chloramphenicol selections, and became less common over time. Because most of the source contigs for these multidrug efflux pumps had high identity to Enterobacteriaceae, it is unlikely that the predominance of multidrug efflux pumps in early infancy is a result of bifidobacteria- or lactobacilli-enriched infantile gut microbial communities. Importantly, our past studies with adult and pediatric human microbiome samples have demonstrated functional capture of numerous resistance genes of Firmicutes, Bacteroidetes, and Actinobacteria origin, without over-representation of Proteobacterial genes [5, 28], suggesting the observed Enterobacteriaceae multidrug efflux pump enrichment signal is not an artifact of cloning bias in our E. coli screening host. Prior studies have shown that Enterobacteriaceae are enriched in the gut microbial communities of young infants and decrease with time $[15,29]$; the observed decrease in chloramphenicol-resistant multidrug efflux pumps during the first year of life in this cohort may be a reflection of maturation of the gut microbial community. Chloramphenicol acetyltransferases were more commonly found in mothers, although they were identified in all infant subjects at some timepoint. This finding of cryptic organisms as an important source of novel chloramphenicol resistance genes is consistent with our prior survey of the antibiotic resistome of healthy children [5].

\section{Conclusions}

This longitudinal study of three pairs of healthy twins and their mothers shows that a diverse gut resistome is present in the first 2 months of life. This confirms our prior hypothesis that multiple antibiotic resistance is present in very young infants [5], and underscores the importance of future study to understand the environmental determinants of resistome establishment in the first weeks of life. Prior studies have shown that gut microbial communities and community-encoded microbial functions of twin siblings are similar to each other $[13,15,20,21]$; our study shows that the gut resistomes of twin pairs also remain similar to each other throughout the first year of life, reinforcing the influence of shared host genetic and environmental variables driving 
resistome development. Infant gut resistomes were no more similar to those of their mothers than to unrelated infants, indicating that factors other than the maternal gut microbial composition are critical for shaping the infant fecal microbiome. Recent work has shown that microbial communities on environmental surfaces in hospitals and homes significantly affect the composition of host-associated microbial communities for humans in those habitats [30,31], and soil microbes have also been found to have resistance genes identical to those in human pathogens [32]. Although antibioticresistant bacteria have been identified on many food products [33-35], 1-2-month-old infants are unlikely to have substantial solid food exposure, and thus environmental surface exposures are more likely to shape the gut resistome of young infants. The association between resistance genes that are shared within and between families suggests a potential role for surveillance of resistance genes within families as a first indicator of resistance genes that are becoming prevalent in the larger community. Infants tended to have resistance to clinically important broad-spectrum $\beta$-lactam antibiotics that their mothers lacked; this, in combination with the dissimilarity between the populations of infant and maternal $\beta$-lactamases, suggests that understanding the factors predisposing infant microbiota to harbor these undesirable $\beta$-lactamases may lead to better understanding of the determinants of widespread broad-spectrum $\beta$ lactamase resistance in human communities. Finally, we describe a developmental progression for a potentially core microbial function of chloramphenicol resistance, which progresses from Enterobacteriaceae multidrug efflux pumps in early infancy to an adult-like population of chloramphenicol acetyltransferases that are often encoded by cryptic microbes, providing new insight into the development of microbiome functions in the early life of the human host.

\section{Methods}

Maternal stools were collected at delivery, and infant fecal samples were collected at 1-2 (baseline), 6-7 (30 days after solid food initiation), and 11 months of age (post-antibiotic exposure). All fecal samples used in this study were collected with informed consent for the St. Louis Neonatal Microbiome Initiative (P.I. Dr. Barbara Warner). This study and the St. Louis Neonatal Microbiome Initiative were approved by Washington University's Institutional Review Board (IRB\# 201205152 and 201105492, respectively). Demographics and clinical metadata were securely stored in a RedCap [36] database. The resistomes encoded by these infant and maternal fecal samples were characterized using functional metagenomic selections $[4,5,32]$. We constructed metagenomic libraries by shotgun cloning one- to five-Kb DNA fragments from the 21 fecal samples
(Additional file 1: Table S1) into a pZE21 plasmid vector, and transforming the plasmids into $E$. coli as previously described [5]. These libraries were screened for resistance against 18 antibiotics representing eight drug classes ( $\beta$-lactams, tetracyclines, aminoglycosides, amphenicols, quinolones, sulfonamides, polymyxins, and cycloserine). Resistance was observed to 16 of the 18 antibiotics tested (Additional file 1: Table S2); resistance-conferring fragments were sequenced, assembled, and annotated using the Parallel Annotation and Reassembly of Functional Metagenomic Selections (PARFuMS) iterative assembly pipeline [32] (see online methods). Predicted proteins were clustered at the $97 \%$ identity level using cd-hit $[17,18]$. Resistance proteins and mobilization elements were identified by a previously described keyword string search of predicted proteins annotated by the PARFuMS computational pipeline [5]. Statistical analysis was performed on these $97 \%$ ID clusters using SAS version 9.3. Analyses on resistance protein clusters were performed using log-linear models assuming a negative binomial distribution of protein cluster counts. Tables of pairwise sample similarity between all samples were calculated using the binary Jaccard distance metric from the QIIME package [37].

\section{Availability of supporting data}

The data reported in this paper are tabulated in Additional file 1 and archived in NCBI Genbank (BioProject ID PRJNA287613).

\section{Additional file}

Additional file 1: Supplementary materials. Table S1. Twin

demographics. Table S2. Antibiotics used for functional selections. Table S3. Log-linear model of multidrug efflux pump protein clusters identified in beta-lactam selections following amoxicillin exposure. Table S4-S5. Log-linear model of within-family and between-family sharing of resistance-associated protein clusters. Table S6. Log-linear model of multidrug efflux pump protein clusters identified in chloramphenicol selections in infants and mothers. Table S7. Log-linear model of multidrug efflux pump protein clusters identified in chloramphenicol selections in infants over time. Table S8-S9. Log-linear model of chloramphenicol acetyltransferase protein clusters identified in chloramphenicol selections in infants over time. Table S10. Log-linear model of within-family sharing, between-family sharing, and appearance of resistance-associated protein clusters at difference points in time. Figure S1. Antibiotic resistance phenotypes. Figure S2. Beta-lactam resistance genotype and phenotype. Figure S3. Twin infant fecal resistomes resemble those of their siblings at each timepoint.

\section{Abbreviations}

Gb: gigabases; kb: kilobases; mgDNA: metagenomic DNA; PARFuMS: parallel annotation and reassembly of functional metagenomic selections.

\section{Competing interests}

The authors declare that they have no competing interests.

\section{Authors' contributions}

AMM designed the study, performed experiments, analyzed data, and wrote the manuscript; SA, BW, and SP performed experiments; MKG analyzed data 
and edited the manuscript; MIN curated study samples; ED and WS analyzed data; PIT and BBW curated samples, designed the study, and edited the manuscript; GD designed the study, analyzed data, and edited the manuscript. All authors read and approved the final manuscript.

\section{Acknowledgements}

We thank members of the Dantas lab for helpful discussions of this work. Research reported in this publication was supported in part by grants to G.D. by the National Institute of General Medical Sciences (NIGMS: http:// www.nigms.nih.gov/), of the National Institutes of Health (NIH) under award number R01GM099538, the March of Dimes Foundation under award number 6-FY12-394, and the Children's Discovery Institute under award number MD-II-2011-117, grants to B.B.W. and P.I.T by the National Institute of Diabetes and Digestive and Kidney Diseases (NIDDK: http://www.niddk.nih.gov/) under award number P30DK052574, and the Children's Discovery Institute under award number MDII_2009-1, and support to P.I.T from the Washington University Biobank Core, and the Melvin E. Carnahan Professorship. A.M.M. was supported by an NIH T32 fellowship (T32 HD043010-08; PI. Dr. Alan Schwartz). M.K.G. is supported by a Mr. and Mrs. Spencer T. Olin Fellowship at Washington University and is a NSF graduate research fellow (award number DGE-11143954). The content is solely the responsibility of the authors and does not necessarily represent the official views of the funding agencies.

\section{Funding}

This study was supported by the National Institutes of Health, Children's Discovery Institute, March of Dimes, and National Science Foundation.

\section{Author details}

${ }^{1}$ Department of Pediatrics, Washington University in St Louis School of Medicine, 660 S. Euclid Avenue, St. Louis, MO 63110, USA. ²Department of Pathology and Immunology, Washington University in St. Louis School of Medicine, 660 S. Euclid Avenue, St. Louis, MO 63110, USA. ${ }^{3}$ Center for Genome Sciences and Systems Biology, Washington University in St. Louis School of Medicine, 4444 Forest Park Boulevard, St. Louis, MO 63108, USA. ${ }^{4}$ Department of Biostatistics, Washington University in St. Louis School of Medicine, 660 S. Euclid Avenue, St. Louis, MO 63110, USA. 'Department of Molecular Microbiology, Washington University in St. School of Medicine, 660 S. Euclid Avenue, St. Louis, MO 63110, USA. 'Department of Biomedical Engineering, Washington University in St. Louis, One Brookings Drive, St. Louis, MO 63130, USA.

\section{Received: 1 April 2015 Accepted: 5 June 2015}

\section{Published online: 25 June 2015}

\section{References}

1. Karami N, Martner A, Enne VI, Swerkersson S, Adlerberth I, Wold AE. Transfer of an ampicillin resistance gene between two Escherichia coli strains in the bowel microbiota of an infant treated with antibiotics. J Antimicrob Chemother. 2007;60(5):1142-5.

2. Shoemaker NB, Vlamakis $H$, Hayes K, Salyers AA. Evidence for extensive resistance gene transfer among Bacteroides spp. and among Bacteroides and other genera in the human colon. Appl Environ Microbiol. 2001;67(2):561-8.

3. Trobos M, Lester CH, Olsen JE, Frimodt-Møller N, Hammerum AM. Natural transfer of sulphonamide and ampicillin resistance between Escherichia coli residing in the human intestine. J Antimicrob Chemother. 2009;63(1):80-6.

4. Sommer MO, Dantas G. Antibiotics and the resistant microbiome. Curr Opin Microbiol. 2011;14(5):556-63.

5. Moore AM, Patel S, Forsberg KJ, et al. Pediatric fecal microbiota harbor diverse and novel antibiotic resistance genes. PLoS One. 2013;8(11), e78822.

6. Alicea-Serrano AM, Contreras M, Magris M, Hidalgo G, Dominguez-Bello MG. Tetracycline resistance genes acquired at birth. Arch Microbiol. 2013;195(6):447-51.

7. de Vries LE, Vallès $Y$, Agersø $Y$, et al. The gut as reservoir of antibiotic resistance: microbial diversity of tetracycline resistance in mother and infant. PLoS One. 2011;6(6), e21644.

8. Z Zhang L, Kinkelaar D, Huang Y, Li Y, Li X, Wang HH. Acquired antibiotic resistance: are we born with it? Appl Environ Microbiol. 2011;77(20):7134-41.

9. Gueimonde M, Salminen S, Isolauri E. Presence of specific antibiotic (tet) resistance genes in infant faecal microbiota. FEMS Immunol Med Microbiol. 2006;48(1):21-5.
10. Fouhy F, Ogilvie LA, Jones BV, et al. Identification of aminoglycoside and $\beta$-lactam resistance genes from within an infant gut functional metagenomic library. PLoS One. 2014;9(9), e108016.

11. Vaz LE, Kleinman KP, Raebel MA, et al. Recent trends in outpatient antibiotic use in children. Pediatrics. 2014;133(3):375-85.

12. Lee GC, Reveles KR, Attridge RT, et al. Outpatient antibiotic prescribing in the United States: 2000 to 2010. BMC Med. 2014;12:96.

13. Palmer C, Bik EM, Digiulio DB, Relman DA, Brown PO. Development of the human infant intestinal microbiota. PLoS Biol. 2007;5(7), e177.

14. Koenig JE, Spor A, Scalfone N, et al. Succession of microbial consortia in the developing infant gut microbiome. Proc Natl Acad Sci U S A. 2011;108 Suppl 1:4578-85.

15. Yatsunenko T, Rey FE, Manary MJ, et al. Human gut microbiome viewed across age and geography. Nature. 2012;486(7402):222-7.

16. Harmsen HJ, Wildeboer-Veloo AC, Raangs GC, et al. Analysis of intestinal flora development in breast-fed and formula-fed infants by using molecular identification and detection methods. J Pediatr Gastroenterol Nutr. 2000;30(1):61-7.

17. Fu L, Niu B, Zhu Z, Wu S, Li W. CD-HIT: accelerated for clustering the nextgeneration sequencing data. Bioinformatics. 2012;28(23):3150-2.

18. Li W, Godzik A. Cd-hit: a fast program for clustering and comparing large sets of protein or nucleotide sequences. Bioinformatics. 2006;22(13):1658-9.

19. Diene SM, Merhej V, Henry M, et al. The rhizome of the multidrug-resistant Enterobacter aerogenes genome reveals how new "killer bugs" are created because of a sympatric lifestyle. Mol Biol Evol. 2013;30(2):369-83.

20. Turnbaugh PJ, Hamady M, Yatsunenko T, et al. A core gut microbiome in obese and lean twins. Nature. 2009;457(7228):480-4.

21. Turnbaugh PJ, Quince C, Faith JJ, et al. Organismal, genetic, and transcriptional variation in the deeply sequenced gut microbiomes of identical twins. Proc Natl Acad Sci U S A. 2010;107(16):7503-8.

22. Makino $\mathrm{H}$, Kushiro A, Ishikawa $\mathrm{E}$, et al. Mother-to-infant transmission of intestinal bifidobacterial strains has an impact on the early development of vaginally delivered infant's microbiota. PLoS One. 2013;8(11), e78331.

23. Gurnee EA, Ndao IM, Johnson JR, et al. Gut colonization of healthy children and their mothers with pathogenic ciprofloxacin-resistant Escherichia coli. J Infect Dis. 2015.

24. Dethlefsen $L$, Huse S, Sogin ML, Relman DA. The pervasive effects of an antibiotic on the human gut microbiota, as revealed by deep 165 rRNA sequencing. PLoS Biol. 2008;6(11), e280.

25. Dethlefsen L, Relman DA. Incomplete recovery and individualized responses of the human distal gut microbiota to repeated antibiotic perturbation. Proc Natl Acad Sci U S A. 2011;108 Suppl 1:4554-61.

26. Forsberg KJ, Patel S, Gibson MK, et al. Bacterial phylogeny structures soil resistomes across habitats. Nature. 2014;509(7502):612-6.

27. Forslund K, Sunagawa S, Coelho LP, Bork P. Metagenomic insights into the human gut resistome and the forces that shape it. Bioessays. 2014;36(3):316-29.

28. Sommer MO, Dantas G, Church GM. Functional characterization of the antibiotic resistance reservoir in the human microflora. Science. 2009;325(5944):1128-31.

29. Vallès Y, Artacho A, Pascual-García A, et al. Microbial succession in the gut: directional trends of taxonomic and functional change in a birth cohort of Spanish infants. PLoS Genet. 2014;10(6), e1004406.

30. Lax S, Smith DP, Hampton-Marcell J, et al. Longitudinal analysis of microbial interaction between humans and the indoor environment. Science. 2014;345(6200):1048-52.

31. Brooks B, Firek BA, Miller CS, et al. Microbes in the neonatal intensive care unit resemble those found in the gut of premature infants. Microbiome. 2014;2(1):1.

32. Forsberg KJ, Reyes A, Wang B, Selleck EM, Sommer MO, Dantas G. The shared antibiotic resistome of soil bacteria and human pathogens. Science. 2012;337(6098):1107-11.

33. Berman HF, Riley LW. Identification of novel antimicrobial resistance genes from microbiota on retail spinach. BMC Microbiol. 2013;13:272.

34. Belmar Campos C, Fenner I, Wiese N, et al. Prevalence and genotypes of extended spectrum beta-lactamases in Enterobacteriaceae isolated from human stool and chicken meat in Hamburg, Germany. Int J Med Microbiol. 2014;304(5-6):678-84.

35. Konya $\mathrm{T}$, Koster B, Maughan $\mathrm{H}$, et al. Associations between bacterial communities of house dust and infant gut. Environ Res. 2014;131:25-30. 
36. Harris PA, Taylor R, Thielke R, Payne J, Gonzalez N, Conde JG. Research electronic data capture (REDCap)-a metadata-driven methodology and workflow process for providing translational research informatics support. J Biomed Inform. 2009;42(2):377-81.

37. Caporaso JG, Kuczynski J, Stombaugh J, et al. QIIME allows analysis of highthroughput community sequencing data. Nat Methods. 2010;7(5):335-6.

Submit your next manuscript to BioMed Central and take full advantage of:

- Convenient online submission

- Thorough peer review

- No space constraints or color figure charges

- Immediate publication on acceptance

- Inclusion in PubMed, CAS, Scopus and Google Scholar

- Research which is freely available for redistribution 